ROME1/1423/06

TIT/HEP-549

hep-th/0602123

\title{
Supersymmetry Breaking by Constant Boundary Superpotentials in Warped Space
}

\author{
Nobuhito Maru ${ }^{(a)}{ }^{1}$, Norisuke Sakai ${ }^{(b)}{ }^{2}$, and Nobuhiro Uekusa ${ }^{(b)} 3$ \\ (a) Dipartimento di Fisica, Università di Roma "La Sapienza" \\ and INFN, Sezione di Roma, P.le Aldo Moro 2, I-00185 Roma, Italy \\ (b) Department of Physics, Tokyo Institute of Technology, Tokyo 152-8551, Japan
}

\begin{abstract}
Supersymmetry breaking by constant (field independent) superpotentials localized at boundaries is studied in a supersymmetric warped space model. We calculate the Kaluza-Klein mass spectrum of the hypermultiplet. We take into account of the radion and the compensator supermultiplets, as well as the bulk mass $c$ for the hypermultiplet. The mass splitting is similar to that of the Scherk-Schwarz supersymmetry breaking (in flat space) for large $|c|$, and has an interesting dependence on the bulk mass parameter $c$. We show that the radius is stabilized by the presence of the constant boundary superpotentials.
\end{abstract}

\footnotetext{
${ }^{1}$ E-mail: Nobuhito.Maru@roma1.infn.it

${ }^{2}$ E-mail: nsakai@th.phys.titech.ac.jp

${ }^{3}$ E-mail: uekusa@th.phys.titech.ac.jp
} 


\section{Introduction}

Supersymmetry (SUSY) [1] is a well-motivated extension to the Standard Model, which plays a crucial role in solving the gauge hierarchy problem. Extra dimensions with flat space 2, 3 or with the warped space 4 are also an alternative solution to the gauge hierarchy problem. Considering both ingredients is natural in the context of the string theory and is often taken as the starting point in the phenomenological model of the brane world scenarios. In such a setup, we have to compactify extra dimensions and break SUSY to obtain realistic four-dimensional physics. One of the simple ways to realize it is the Scherk-Schwarz (SS) mechanism of SUSY breaking [5].

It is known that the SS SUSY breaking is equivalent to the SUSY breaking by a (bulk) constant (field independent) superpotential in flat space [6, 7, 8]. These two scenarios generate the same mass spectrum. It is natural to ask whether this equivalence still holds in warped space. This issue has been discussed in several interesting papers so far 9. 10, 11]. According to [9], if one considers the gauging of a symmetry of the theory in warped space, which is completely broken by the boundary conditions, one is led to an inconsistent theory. This implies an inconsistency of the SS SUSY breaking in a SUSY Randall-Sundrum model. In [10], they discuss the SS twist for $S U(2)_{R}$ in the fivedimensional gauged supergravity. Their conclusion is that if the background geometry is $A d S_{4}$, SUSY is broken by the SS twist, but if the background geometry is the RandallSundrum geometry, SUSY is not broken by the SS twist. This statement agrees with that of [9]. Recently, [11] also discussed this issue from the viewpoint of the five-dimensional conformal supergravity. It tells us that whether SUSY is broken by the SS twist or not in warped space depends on the way to gauge $U(1)_{R}$. If we gauge $U(1)_{R}$ by the graviphoton with $Z_{2}$-odd gauge coupling, SUSY is not broken by the SS twist. However, they pointed out that if we gauge $U(1)_{R}$ by the graviphoton with $Z_{2}$-even gauge coupling, it is possible to break SUSY. This seems to disagree with the statement in 9, 10. In the light of these facts, the issue of the SS SUSY breaking in warped space is not settled. Furthermore, the equivalence of the SS SUSY breaking to the SUSY breaking by a constant (field independent) superpotential in warped space is also unclear compared to the flat space case. A number of works have studied the SUSY Randall-Sundrum model [12, 13, 14, 15.

More recently, higher dimensional gauge theories are also used to provide Higgs fields as an extra-dimensional component of gauge fields [16. The true ground state of such a system is generally determined by the one-loop effective potential [17]-[20, which requires mass spectrum of all the Kaluza-Klein towers, especially their SUSY breaking pattern, since the effective potential vanishes in the supersymmetric limit. For phenomenological applications, it is also important to include bulk mass parameters for hypermultiplets [20].

The purpose of this paper is to investigate SUSY breaking effects and its properties caused by constant (field independent) superpotentials localized at fixed points in the SUSY Randall-Sundrum model. Taking the hypermultiplet and including the compensating multiplet and the radion multiplet consistently (from supergravity), we calculate the Kaluza-Klein mass spectrum and their mode functions of the hypermultiplet. We find that the mass spectrum depends on the bulk mass parameter in addition to the strength of the constant boundary superpotential. We observe a similarity of our result to the SS SUSY breaking spectrum in flat space for large bulk mass parameter $|c|$. We also show 
that the radius is stabilized by the presence of the constant boundary superpotentials ${ }^{4}$.

This paper is organized as follows. The model is introduced in Sec. The mass spectrum of the hypermultiplet is calculated in Sec 3 and is compared with the SS SUSY breaking. The mode functions are obtained in Sec4. We show in Sec 5 that the radius is stabilized. Sec 6 gives a conclusion. Appendices include a few details of calculations.

\section{Model}

We consider a five-dimensional supersymmetric model of a single hypermultiplet on the Randall-Sundrum background, whose metric is

$$
d s^{2}=e^{-2 R \sigma} \eta_{\mu \nu} d x^{\mu} d x^{\nu}+R^{2} d y^{2}, \quad \sigma(y) \equiv k|y|,
$$

where $R$ is the radius of $S^{1}$ of the orbifold $S^{1} / Z_{2}, k$ is the $A d S_{5}$ curvature scale, and the angle of $S^{1}$ is denoted by $y(0 \leq y \leq \pi)$. In terms of superfields for four manifest supersymmetry, our Lagrangian reads [6]

$$
\begin{aligned}
\mathcal{L}_{5}= & \int d^{4} \theta \frac{1}{2} \varphi^{\dagger} \varphi\left(T+T^{\dagger}\right) e^{-\left(T+T^{\dagger}\right) \sigma}\left(\Phi^{\dagger} \Phi+\Phi^{c} \Phi^{c \dagger}-6 M_{5}^{3}\right) \\
& +\int d^{2} \theta\left[\varphi^{3} e^{-3 T \sigma}\left\{\Phi^{c}\left[\partial_{y}-\left(\frac{3}{2}-c\right) T \sigma^{\prime}\right] \Phi+W_{b}\right\}+\text { h.c. }\right]
\end{aligned}
$$

where $\varphi=1+\theta^{2} F_{\varphi}$ is the compensator chiral supermultiplet (of supergravity), $T=R+$ $\theta^{2} F_{T}$ is the radion chiral supermultiplet, and $\Phi, \Phi^{c}$ are chiral supermultiplets representing the hypermultiplet. The $Z_{2}$ parity is assigned to be even (odd) for $\Phi\left(\Phi^{c}\right)$. The derivative with respect to $y$ is denoted by ', such as $\sigma^{\prime} \equiv d \sigma / d y$. The five-dimensional Planck mass is denoted as $M_{5}$. Here we consider a model with constant (field independent) superpotentials localized at the fixed points $y=0, \pi$

$$
W_{b} \equiv 2 M_{5}^{3}\left(w_{0} \delta(y)+w_{\pi} \delta(y-\pi)\right)
$$

where $w_{0}, w_{\pi}$ are dimensionless constants which will be assumed to be $\mathcal{O}(1)$.

Since interesting physics in the extra dimensions is contained solely in the part of the Lagrangian (2.2) containing auxiliary components $F, F^{c}, F_{T}, F_{\varphi}$, we extract that part

$$
\begin{aligned}
\mathcal{L}_{\text {aux }}= & \left(\frac{1}{2} e^{-2 R \sigma}\left(2 R F^{\dagger} F+F_{T} F^{\dagger} \phi+F_{T}^{\dagger} F \phi^{\dagger}\right)\right. \\
& \left.+\left\{\frac{1}{2} e^{-2 R \sigma}\left(2 R \phi^{\dagger} F+F_{T}\left(\phi^{\dagger} \phi-3 M_{5}^{3}\right)\right)\left(F_{\varphi}^{\dagger}-F_{T}^{\dagger} \sigma\right)+\text { h.c. }\right\}+\left(\phi \leftrightarrow \phi^{c}\right)\right) \\
& +e^{-2 R \sigma} R\left(\phi^{\dagger} \phi+\phi^{c} \phi^{c \dagger}-6 M_{5}^{3}\right)\left(F_{\varphi}^{\dagger}-F_{T}^{\dagger} \sigma\right)\left(F_{\varphi}-F_{T} \sigma\right) \\
& +\left[3 e^{-3 R \sigma}\left(F_{\varphi}-F_{T} \sigma\right)\left\{\phi^{c}\left[\partial_{y}-\left(\frac{3}{2}-c\right) R \sigma^{\prime}\right] \phi+W_{b}\right\}\right. \\
& +e^{-3 R \sigma}\left\{F^{c}\left[\partial_{y}-\left(\frac{3}{2}-c\right) R \sigma^{\prime}\right] \phi+\phi^{c}\left[\partial_{y}-\left(\frac{3}{2}-c\right) R \sigma^{\prime}\right] F\right. \\
& \left.\left.-\phi^{c}\left(\frac{3}{2}-c\right) F_{T} \sigma^{\prime} \phi\right\}+ \text { h.c. }\right],
\end{aligned}
$$

\footnotetext{
${ }^{4}$ For related discussions on stabilization, see 21, 22, 23, for example.
} 
where $\phi^{c}$ and $F^{c}(\phi$ and $F)$ are scalar and auxiliary components of $\Phi^{c}(\Phi)$. The Lagrangian (2.4) gives the following equations of motion for auxiliary fields

$$
\begin{aligned}
F= & -\frac{e^{-R \sigma}}{R}\left[-\partial_{y} \phi^{c \dagger}+\left(\frac{3}{2}+c\right) R \sigma^{\prime} \phi^{c \dagger}+\frac{\phi}{2 M_{5}^{3}} W_{b}\right. \\
& \left.+\frac{1}{6 M_{5}^{3}} \phi^{\dagger} \phi \partial_{y} \phi^{c \dagger}+\frac{1}{3 M_{5}^{3}} \phi^{c \dagger} \phi \partial_{y} \phi^{\dagger}-\frac{1}{6 M_{5}^{3}} \phi^{\dagger} \phi \phi^{c \dagger}\left(\frac{9}{2}-c\right) R \sigma^{\prime}\right] \\
F^{c}= & -\frac{e^{-R \sigma}}{R}\left[\partial_{y} \phi^{\dagger}-\left(\frac{3}{2}-c\right) R \sigma^{\prime} \phi^{\dagger}+\frac{\phi^{c}}{2 M_{5}^{3}} W_{b}\right. \\
& \left.+\frac{1}{6 M_{5}^{3}} \phi^{c} \phi^{\dagger} \partial_{y} \phi^{c \dagger}+\frac{1}{3 M_{5}^{3}} \phi^{c \dagger} \phi^{c} \partial_{y} \phi^{\dagger}-\frac{1}{6 M_{5}^{3}} \phi^{c} \phi^{\dagger} \phi^{c \dagger}\left(\frac{9}{2}-c\right) R \sigma^{\prime}\right] \\
F_{\varphi}=- & \frac{e^{-R \sigma}}{R}\left[-\frac{1}{6 M_{5}^{3}} \phi^{\dagger} \partial_{y} \phi^{c \dagger}-\frac{1}{3 M_{5}^{3}} \phi^{c \dagger} \partial_{y} \phi^{\dagger}+\frac{1}{6 M_{5}^{3}} \phi^{\dagger} \phi^{c \dagger}\left(\frac{9}{2}-c\right) R \sigma^{\prime}-\frac{1}{2 M_{5}^{3}} W_{b}\right. \\
& \left.-\frac{3(1-2 R \sigma)}{r} \phi^{c \dagger} \partial_{y} \phi^{\dagger}-\frac{3(1-2 R \sigma)}{r} W_{b}+\frac{1-2 R \sigma}{r} \phi^{c \dagger} \phi^{\dagger}\left(\frac{3}{2}-c\right) R \sigma^{\prime}\right] \\
F_{T}= & -\frac{e^{-R \sigma}}{r}\left[6 \phi^{c \dagger} \partial_{y} \phi^{\dagger}-2 \phi^{c \dagger} \phi^{\dagger}\left(\frac{3}{2}-c\right) R \sigma^{\prime}+6 W_{b}\right]
\end{aligned}
$$

where the partial integration has been performed in (2.5) and $r \equiv \phi^{\dagger} \phi+\phi^{c \dagger} \phi^{c}-6 M_{5}^{3}$. We can eliminate these auxiliary fields in (2.4) by substituting (2.5) (2.8). To obtain the mass spectrum, we take out only the part of the Lagrangian which is bilinear in scalar fields

$$
\begin{aligned}
& \mathcal{L}_{\text {aux }}^{\text {bilinear }}=-\frac{1}{R} e^{-4 R \sigma}\left[\left|-\partial_{y} \phi^{c \dagger}+\left(\frac{3}{2}+c\right) R \sigma^{\prime} \phi^{c \dagger}+\frac{\phi}{2 M^{3}} W_{b}\right|^{2}\right. \\
& \left.+\left|\partial_{y} \phi^{\dagger}-\left(\frac{3}{2}-c\right) R \sigma^{\prime} \phi^{\dagger}+\frac{\phi^{c}}{2 M^{3}} W_{b}\right|^{2}+\left\{\frac{W_{b}}{M^{3}}\left(\frac{3}{2}-c\right) R \sigma^{\prime} \phi \phi^{c}+\text { h.c. }\right\}\right] .
\end{aligned}
$$

The bilinear part of the Lagrangian turns out to be identical to that derived from another Lagrangian

$$
\begin{aligned}
\mathcal{L}_{5} & =\int d^{4} \theta \frac{1}{2} \varphi^{\dagger} \varphi\left(T+T^{\dagger}\right) e^{-2 R \sigma}\left(\Phi^{\dagger} \Phi+\Phi^{c} \Phi^{c \dagger}-6 M_{5}^{3}\right) \\
& +\int d^{2} \theta\left[\varphi^{2} e^{-3 R \sigma}\left\{\Phi^{c}\left[\partial_{y}-\left(\frac{3}{2}-c\right) T \sigma^{\prime}\right] \Phi+W\right\}+\text { h.c. }\right],
\end{aligned}
$$

which was proposed in Ref. 24] based on a more accurate treatment of the radion superfield using supergravity.

\section{Mass spectrum of hyperscalar}

Let us calculate the mass spectrum of scalar component fields $\phi$ and $\phi^{c}$ of the hyper-

multiplet. To allow possible discontinuities of the $Z_{2}$ odd field $\phi^{c}$ across the fixed points $y=0, \pi$, we define

$$
\phi^{c}(x, y) \equiv \hat{\epsilon}(y) h^{c}(x, y), \quad \hat{\epsilon}(y) \equiv\left\{\begin{array}{l}
+1, \quad 0<y<\pi \\
-1, \quad-\pi<y<0
\end{array},\right.
$$


where $h^{c}(x, y)$ is a parity even function with possibly nonvanishing value at $y=0, \pi$.

The equations of motion for $\phi$ and $\phi^{c}$ are given by

$$
\begin{aligned}
0= & \frac{W_{b}}{2 M_{5}^{3}}\left(-2(\delta(y)-\delta(y-\pi)) h^{c}+\frac{\phi^{\dagger}}{2 M_{5}^{3}} W_{b}+7(\hat{\epsilon}(y))^{2} k R h^{c}-\hat{\epsilon}(y) \partial_{y} h^{c}\right) \\
& -R^{2} e^{2 R \sigma} \eta^{\mu \nu} \partial_{\mu} \partial_{\nu} \phi^{\dagger}-e^{\left(\frac{5}{2}+c\right) R \sigma} \partial_{y}\left(e^{-(1+2 c) R \sigma} \partial_{y}\left(e^{-\left(\frac{3}{2}-c\right) R \sigma} \phi^{\dagger}\right)\right), \\
0= & \frac{W_{b}}{2 M^{3}}\left(\frac{\hat{\epsilon}(y) h^{c \dagger}}{2 M^{3}} W_{b}+2 \partial_{y} \phi\right)-2(2 \delta(y)-2 \delta(y-\pi)) \partial_{y} h^{c \dagger} \\
- & 2\left(\partial_{y} \delta(y)-\partial_{y} \delta(y-\pi)\right) h^{c \dagger}+\frac{\phi}{2 M^{3}} \partial_{y} W_{b} \\
+ & \hat{\epsilon}(y)\left[-R^{2} e^{2 R \sigma} \eta^{\mu \nu} \partial_{\mu} \partial_{\nu} h^{c \dagger}-e^{\left(\frac{5}{2}-c\right) R \sigma} \partial_{y}\left(e^{-(1-2 c) R \sigma} \partial_{y}\left(e^{-\left(\frac{3}{2}+c\right) R \sigma} h^{c \dagger}\right)\right)\right] .
\end{aligned}
$$

One should note that the equations for $\phi$ and $\phi^{c}$ couple only through the boundary superpotential $W_{b}$. In the limit of vanishing boundary superpotential, we should obtain a series of effective fields with $\phi(x, y)$ component only, and another series of effective fields with $\phi^{c}(x, y)$ component only. For nonvanishing boundary superpotential, these two sets mix each other and we obtain $n$-th Kaluza-Klein effective field $\phi_{n}^{I}(x)$ with its mode functions $b_{n}^{I}(y)$ as $\phi(x, y)$ component and $b_{n}^{c I}(y)$ as $\phi^{c}(x, y)$ component

$$
\left(\begin{array}{c}
\phi(x, y) \\
\phi^{c}(x, y)
\end{array}\right)=\sum_{n} \sum_{I=1,2} \phi_{n}^{I}(x)\left(\begin{array}{c}
b_{n}^{I}(y) \\
\hat{\epsilon}(y) b_{n}^{c I}(y)
\end{array}\right),
$$

where $I$ is the indices corresponding to the two independent effective fields eigenvalues.

Assuming that the effective four-dimensional field $\phi_{n}(x)$ has mass $m_{n}$, we easily find solutions in the bulk in terms of the Bessel functions $J_{\alpha},\left(J_{\beta}\right)$ and $Y_{\alpha},\left(Y_{\beta}\right)[13$

$$
\begin{array}{ll}
b_{n}(y)=\frac{e^{2 R \sigma}}{N_{n}}\left[J_{\alpha}\left(m_{n} e^{R \sigma} / k\right)+b_{\alpha}\left(m_{n}\right) Y_{\alpha}\left(m_{n} e^{R \sigma} / k\right)\right], \quad \alpha=\left|c+\frac{1}{2}\right|, \\
b_{n}^{c}(y)=\frac{e^{2 R \sigma}}{N_{n}^{c}}\left[J_{\beta}\left(m_{n} e^{R \sigma} / k\right)+b_{\beta}\left(m_{n}\right) Y_{\beta}\left(m_{n} e^{R \sigma} / k\right)\right], \quad \beta=\left|c-\frac{1}{2}\right|,
\end{array}
$$

where we have not yet specified which mode $I=1,2$ until we determine mass eigenvalues later.

Because of $\delta(y), \delta(y-\pi)$ in the boundary superpotential and also derivatives of sign function $\hat{\epsilon}(y)$, we obtain several types of singular contributions, $\delta^{2}(y)$ terms, $\partial_{y} \delta(y)$ terms and $\delta(y)$ terms and similarly for $y$ replaced by $y-\pi$. To find out these singular terms, we use the following identity valid as a result of a properly regularized calculation ${ }^{5}$

$$
\delta(y)(\hat{\epsilon}(y))^{2}=\frac{1}{3} \delta(y), \quad \delta(y-\pi)(\hat{\epsilon}(y))^{2}=\frac{1}{3} \delta(y-\pi) .
$$

All these singular terms need to be canceled resulting in boundary conditions. The first boundary condition comes from $\delta^{2}$ terms in the equation of motion (3.2) for $\phi$

$$
\begin{aligned}
-2 b_{n}^{c}(0)+w_{0} b_{n}(0) & =0, \\
2 b_{n}^{c}(\pi)+w_{\pi} b_{n}(\pi) & =0 .
\end{aligned}
$$

\footnotetext{
${ }^{5}$ This follows from a wide range of regularization respecting the relation $2 \delta(y)=d \epsilon(y) / d y$.
} 
The second boundary condition comes from $\delta$ function in the equation of motion for $\phi$

$$
\begin{aligned}
0 & =\frac{7}{3} w_{0} b_{n}^{c}(0)-\frac{1}{3} w_{0}\left[2 b_{n}^{c}(0)+\frac{m_{n}}{k} \frac{1}{N_{n}^{c}}\left\{J_{\beta}^{\prime}\left(m_{n} / k\right)+b_{\beta} Y_{\beta}^{\prime}\left(m_{n} / k\right)\right\}\right] \\
& -4 b_{n}(0)+2\left(\frac{3}{2}-c\right) b_{n}(0)-\frac{2 m_{n}}{k}\left[\frac{1}{N_{n}}\left\{J_{\alpha}^{\prime}\left(m_{n} / k\right)+b_{\alpha}\left(m_{n}\right) Y_{\alpha}^{\prime}\left(m_{n} / k\right)\right\}\right], \\
0 & =\frac{7}{3} w_{\pi} b_{n}^{c}(\pi)-\frac{1}{3} w_{\pi}\left[2 b_{n}^{c}(\pi)+\frac{m_{n}}{k} \frac{e^{3 R k \pi}}{N_{n}^{c}}\left\{J_{\beta}^{\prime}\left(m_{n} e^{R k \pi} / k\right)+b_{\beta} Y_{\beta}^{\prime}\left(m_{n} e^{R k \pi} / k\right)\right\}\right] \\
& +4 b_{n}(\pi)-2\left(\frac{3}{2}-c\right) b_{n}(\pi)+\frac{2 m_{n}}{k}\left[\frac{e^{3 R k \pi}}{N_{n}}\left\{J_{\alpha}^{\prime}\left(m_{n} e^{R k \pi} / k\right)+b_{\alpha}\left(m_{n}\right) Y_{\alpha}^{\prime}\left(m_{n} e^{R k \pi} / k\right)\right\}\right]
\end{aligned}
$$

with $J^{\prime}(z)=d J(z) / d z$. We find that no additional boundary conditions ${ }^{6}$ arises from the equation of motion of $\phi^{c}$, since the boundary condition from $\partial_{y} \delta$ is identical to that from $\delta^{2}$.

We wish to solve the boundary conditions (3.8)-(3.11) in the limit

$$
\begin{gathered}
m_{n} / k \ll 1, \\
m_{n} e^{k R \pi} / k \gg 1 .
\end{gathered}
$$

Because of (3.12), the coefficient $b_{\alpha}$ and $b_{\beta}$ is small and $Y_{\alpha}, Y_{\beta}$ terms can be neglected at $y=\pi$. As given in Appendix A, the condition (3.13) allows the remaining Bessel functions $J_{\alpha}$ and $J_{\beta}$ to be approximated by their asymptotic forms.

After changing variables,

$$
\frac{m_{n}}{k} e^{R k \pi} \equiv x, \quad \frac{2 \alpha+1}{4} \pi \equiv a, \quad \frac{2 \beta+1}{4} \pi \equiv b,
$$

we can rewrite boundary conditions at $y=\pi$ (A.10) and A.11 as summarized in Appendix $\mathrm{A}$ into a matrix form

$$
\begin{aligned}
{\left[\begin{array}{ll}
M_{11} & M_{12} \\
M_{21} & M_{22}
\end{array}\right]\left[\begin{array}{l}
1 / N_{n}^{c} \\
1 / N_{n}
\end{array}\right]=0 . } \\
M_{11}=2 \cos (x-b), \\
M_{12}=w_{\pi} \cos (x-a), \\
M_{21}=\frac{w_{\pi}}{6}\left(\left(c^{2}-c+11\right) \cos (x-b)+2 x \sin (x-b)\right), \\
M_{22}=(c(1-c) \cos (x-a)-2 x \sin (x-a)) .
\end{aligned}
$$

Mass spectrum is determined by nontrivial solutions of fluctuations require the vanishing determinant of the matrix $M$

$$
\begin{aligned}
0= & 2 \cos (x-b)[c(1-c) \cos (x-a)-2 x \sin (x-a)] \\
& -w_{\pi}^{2} \cos (x-a)\left(\frac{c^{2}-c+11}{6} \cos (x-b)+\frac{x}{3} \sin (x-b)\right),
\end{aligned}
$$

\footnotetext{
${ }^{6}$ Other potentially singular contributions do not contribute because of $\hat{\epsilon} \times \delta=0, \hat{\epsilon} \times \delta^{2}=0$.
} 
which determines the mass $m_{n}$. It is most useful to start from the limit of vanishing boundary superpotential, $w_{\pi} \rightarrow 0$, giving two possibilities. The first solution is purely $\phi(x, y)$ modes $\left(\phi^{c}(x, y)=0\right)$

$$
0=c(1-c) \cos (x-a)-2 x \sin (x-a),
$$

which gives solutions for large $x$ as

$$
x=n \pi+a+\frac{c(1-c)}{2 n \pi}+\mathcal{O}\left(\frac{1}{n^{2}}\right)
$$

with integer $n$. The second solution is purely $\phi^{c}(x, y)$ modes $(\phi(x, y)=0)$

$$
0=\cos (x-b)
$$

which gives solutions

$$
x=\left(n-\frac{1}{2}\right) \pi+b
$$

with integer $n$. When $w_{\pi} \neq 0$, these two modes (3.22) and (3.24) mix each other to form two independent modes with slightly different mass spectra and with associated mode functions.

We can rewrite the eigenvalue equation (3.20) in a more convenient form

$$
\begin{gathered}
\tan ^{2}(x-a)-A(x) \tan (x-a)+B(x)=0 \\
A(x) \equiv \frac{C}{2 x}+\left(1+\frac{w_{\pi}^{2}}{12}\right) \cot (a-b), \quad B(x) \equiv \frac{C}{2 x} \cot (a-b)-\frac{w_{\pi}^{2}}{12} \\
C \equiv c(1-c)-\frac{\left(c^{2}-c+11\right) w_{\pi}^{2}}{12}
\end{gathered}
$$

Since we are interested in highly excited Kaluza-Klein states $(n \gg 1)$, we can approximate $x \approx n \pi$ in the denominator of $C /(2 x)$. Thus we find

$$
\tan (x-a) \approx \frac{A(n \pi)}{2}\left(1 \pm \sqrt{1-\frac{4 B(n \pi)}{A^{2}(n \pi)}}\right)
$$

We can determine the sign choice in (3.28) by examining the solution near $w_{\pi} \rightarrow 0$. Since the upper (lower) sign for $0 \leq c \leq 1(c<0,1<c)$ reduces to the behavior in Eq.(3.22) at $w_{\pi} \rightarrow 0$, it should correspond to the mode reducing to the purely $\phi(x, y)$ component at $w_{\pi} \rightarrow 0$. We shall call this mode as the dominantly $\phi$ mode, and denote it by the mode functions $(I=1)$ as $b_{n}^{1}(y), b_{n}^{c 1}(y)$ and the effective field as $\phi_{n}^{I=1}(x) \equiv \phi_{n}(x)$ in equation (3.4). The other choice of the sign corresponds to the mode reducing to the purely $\phi^{c}(x, y)$ component at $w_{\pi} \rightarrow 0$, which behaves as in Eq.(3.24). We shall call this mode as the dominantly $\phi^{c}$ mode, and denote the mode functions $(I=2)$ as $b_{n}^{2}(y), b_{n}^{c 2}(y)$ and the effective field as $\phi_{n}^{I=2}(x) \equiv \phi_{n}^{c}(x)$ in equation (3.4).

Dominantly $\phi$ mode: $\phi^{I=1} \equiv \phi$ 
We first consider the dominantly $\phi$ mode. Depending on the value of the bulk mass parameter $c$, there is a qualitative difference of mass spectrum as a function of $w_{\pi}$. Let us first consider the case of $|c| \geq 1 / 2$. Then the parameter becomes $a-b= \pm \pi / 2$, which makes the energy levels for purely $\phi$ and $\phi^{c}$ modes to be split only small amount of the order of $\mathcal{O}(1 / n \pi)$ at high enough excitation $(n \gg 1)$. Therefore we need to distinguish two cases depending on the relative magnitude of $\left|w_{\pi}\right|$ and $1 /(n \pi)$. For $\left|w_{\pi}\right| \ll 1 /(n \pi) \ll 1$, we obtain perturbation from nondegenerate eigenvalues to find a deviation proportional to $w_{\pi}^{2}$

$$
\tan (x-a) \approx \frac{c(1-c)}{2 n \pi}\left[1+\frac{1}{3}\left(\frac{n \pi w_{\pi}}{c(1-c)}\right)^{2}\right] .
$$

For $w_{\pi} \sim \mathcal{O}(1)$, we obtain perturbation from (approximately) degenerate eigenvalues to find a deviation linear in $w_{\pi}$

$$
\tan (x-a) \approx \pm \frac{\left|w_{\pi}\right|}{2 \sqrt{3}}\left\{\begin{array}{l}
+ \text { for } 1 / 2 \leq c \leq 1 \\
- \text { for } c \leq-1 / 2 \text { or } c>1
\end{array},\right.
$$

which gives the mass

$$
m_{n} \approx k e^{-R k \pi}\left[\left(n+\frac{2 \alpha+1}{4}\right) \pi \pm \frac{\left|w_{\pi}\right|}{2 \sqrt{3}}\right], \quad w_{\pi} \sim \mathcal{O}(1)
$$

where the plus (minus) sign should be taken for $1 / 2 \leq c \leq 1(c \leq-1 / 2$ or $c>1)$.

We show $\tan (x-a)$ with the upper sign (dominantly $\phi$ mode) as a function of $w_{\pi}$ in Fig. 1.

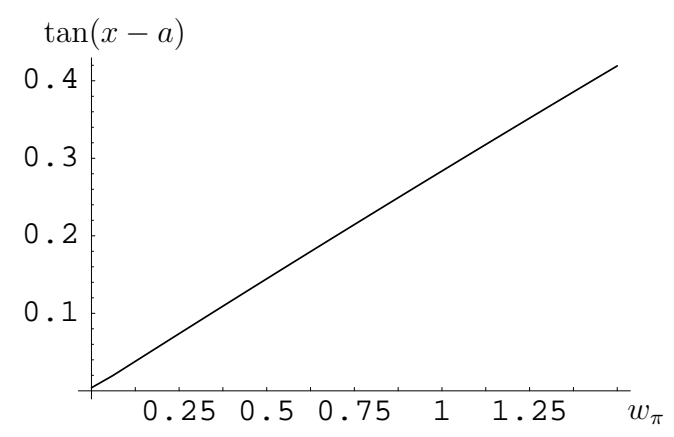

Figure 1: The $w_{\pi}$ dependence of $\tan (x-a)$ with the upper sign (dominantly $\phi$ mode) in the case of $|c| \geq 1 / 2$. We use $c=0.5$ and $n=10$.

For the case of $|c|<1 / 2$, the parameter becomes $a-b=c \pi$, which makes the energy levels for purely $\phi$ and $\phi^{c}$ modes to be nondegenerate even at high excitation $(n \gg 1)$. Therefore we always obtain a mass shift proportional to $w_{\pi}^{2}$. For $w_{\pi} \ll 1 /(n \pi)$, the solution is approximated by

$$
\tan (x-a) \approx \frac{c(1-c)}{2 n \pi}-\frac{w_{\pi}^{2} \tan c \pi}{12} .
$$

For $w_{\pi} \sim \mathcal{O}(1)$, it is approximated by

$$
\tan (x-a) \approx \frac{1}{2 \tan c \pi}\left(1+\frac{w_{\pi}^{2}}{12}\right)\left(1-\sqrt{1+\frac{w_{\pi}^{2}}{3} \tan ^{2} c \pi}\right) .
$$


This gives the mass whose $w_{\pi}$ dependence involves the bulk mass parameter $c$

$$
m_{n} \approx k e^{-R k \pi}\left[\left(n+\frac{2 \alpha+1}{4}\right) \pi+\frac{w_{\pi}^{2}+12}{24 \tan c \pi}\left(1-\sqrt{1+\frac{w_{\pi}^{2}}{3} \tan ^{2} c \pi}\right)\right] .
$$

We show $\tan (x-a)$ as a function of $w_{\pi}$ in Fig 2 ,

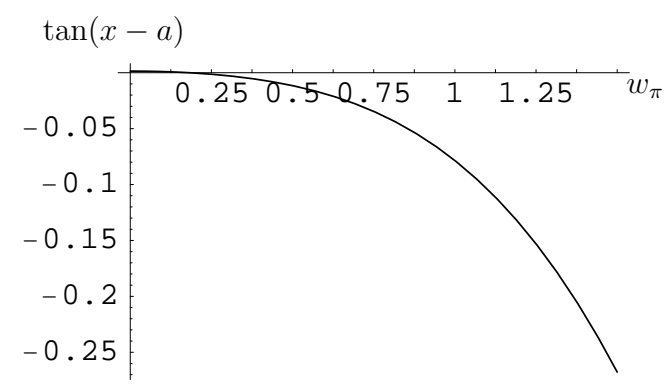

Figure 2: The $w_{\pi}$ dependence of $\tan (x-a)$ with the upper sign (dominantly $\phi$ mode) in the case of $|c|<1 / 2$. We use $c=0.1$ and $n=10$.

Dominantly $\phi^{c}$ mode: $\phi^{I=2} \equiv \phi^{c}$

If we choose the lower sign in Eq.(3.28), we obtain the dominantly $\phi^{c}$ mode. In the case of $|c| \geq 1 / 2$, we show $\tan (x-a)$ as a function of $w_{\pi}$ in Fig [3. For $w_{\pi} \ll 1 /(n \pi) \ll 1$

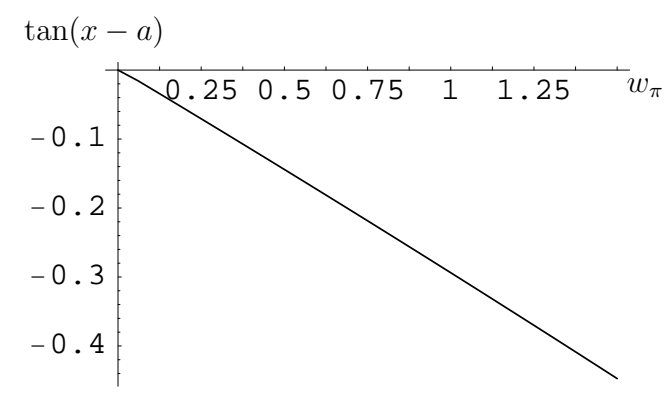

Figure 3: The $w_{\pi}$ dependence of $\tan (x-a)$ with the lower sign (dominantly $\phi^{c}$ mode) in the case of $|c| \geq 1 / 2$. We use $c=0.5$ and $n=10$.

in the case of $|c| \geq 1 / 2$, we obtain

$$
\tan (x-a) \approx-\frac{n \pi}{6 c(1-c)} w_{\pi}^{2} .
$$

For $w_{\pi} \sim \mathcal{O}(1)$ in the case of $|c| \geq 1 / 2$, it becomes

$$
\tan (x-a) \approx \mp \frac{\left|w_{\pi}\right|}{2 \sqrt{3}}\left\{\begin{array}{l}
- \text { for } \quad 1 / 2 \leq c \leq 1 \\
+ \text { for } c \leq-1 / 2 \text { or } c>1
\end{array}\right.
$$

which gives the mass

$$
m_{n} \approx k e^{-R k \pi}\left[\left(n-\frac{1}{2}+\frac{2 \beta+1}{4}\right) \pi \mp \frac{\left|w_{\pi}\right|}{2 \sqrt{3}}\right](n \gg 1) .
$$




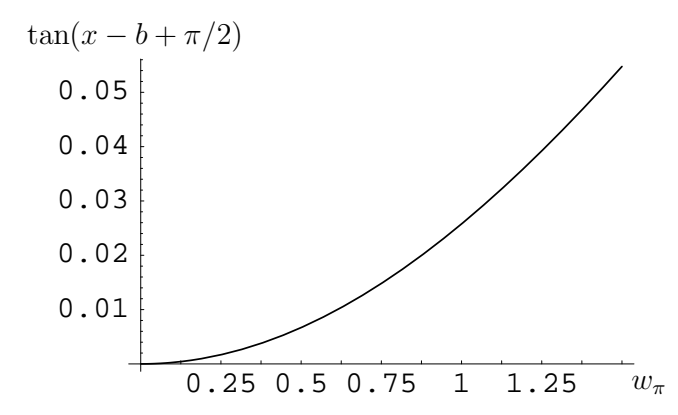

Figure 4: The $w_{\pi}$ dependence of $\tan (x-b+\pi / 2)$ for dominantly $\phi^{c}$ mode in the case of $|c|<1 / 2$. We use $c=0.1$ and $n=10$.

where the plus (minus) sign should be chosen for $c \leq-1 / 2$ or $c>1(1 / 2 \leq c \leq 1)$.

In the case of $|c|<1 / 2$, we show $\tan (x-b+\pi / 2)$ instead of $\tan (x-a)$ in Fig 4 . It is approximated as

$$
\tan (x-b+\pi / 2) \approx \frac{w_{\pi}^{2} \tan c \pi}{12}
$$

which gives the mass whose $w_{\pi}$ dependence involves the bulk mass parameter $c$

$$
m_{n} \approx k e^{-R k \pi}\left[\left(n-\frac{1}{2}+\frac{2 \beta+1}{4}\right) \pi+\frac{w_{\pi}^{2} \tan c \pi}{12}\right] .
$$

As for the mass spectrum of hyperfermions, one can immediately check that the linearized equations of motion for hyperfermions are not affected by the constant boundary superpotentials $w_{0}, w_{\pi}$ localized at branes. Therefore the mass spectrum for hyperfermions is that for hyperscalars with $w_{\pi}=0$.

\section{Mode functions}

Let us determine the mode functions for each eigenvalue obtained above. The corresponding eigenvector of each solution gives the ratio $N_{n} / N_{n}^{c}$ for the mode functions $\left(b_{n}, \hat{\epsilon} b_{n}^{c}\right)^{T}$ in Eqs. (3.4)-(3.6).

Dominantly $\phi$ mode: $\phi^{I=1} \equiv \phi$

We first consider the dominantly $\phi$ modes corresponding to the choice of the upper sign in Eq.(3.28). Using (3.15) -(3.19), we find the eigenvector

$$
\frac{N_{n}}{N_{n}^{c}}=-\frac{M_{12}}{M_{11}}=-\frac{M_{22}}{M_{21}}
$$

which is most easily evaluated by

$$
\frac{M_{12}}{M_{11}}=\frac{w_{\pi}}{2} \frac{1}{\cos (a-b)-\tan (x-a) \sin (a-b)},
$$

For $|c| \leq 1 / 2$, we obtain the ratio $N_{n} / N_{n}^{c}$ which is shown as a function of $w_{\pi}$ in Fig 5 , using the solution (3.28) with the upper sign for the dominantly $\phi$ modes. Using (3.32) 


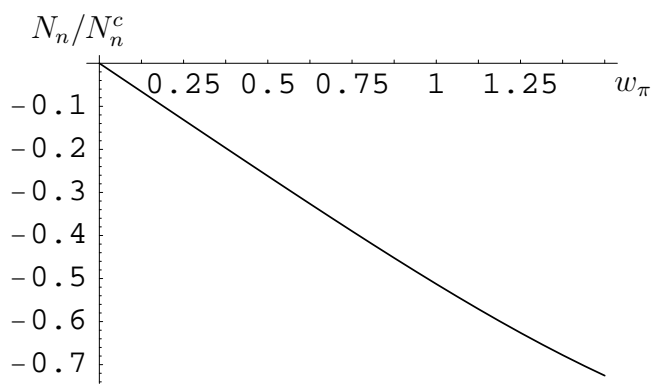

Figure 5: The ratio $N_{n} / N_{n}^{c}$ as a function $w_{\pi}$ in the case of $|c|<1 / 2$. We use $c=0.1$ and $n=10$.

for small $w_{\pi}$, it can be approximated as

$$
\frac{N_{n}}{N_{n}^{c}} \approx-\frac{w_{\pi}}{2 \cos c \pi} .
$$

This result represents $\mathcal{O}\left(w_{\pi}\right)$ mixing of $b_{n}^{c}$ as we expected.

For $|c| \geq 1 / 2$, we obtain the ratio by letting $a-b= \pm \pi / 2$ in Eq.(4.2). Using the solution (3.28) with the upper sign for the dominantly $\phi$ modes, we show $N_{n} / N_{n}^{c}$ as a function of $w_{\pi}$ in Fig 6 ] Using (3.35) for $w \ll(1 / n \pi)$, the ratio $N_{n} / N_{n}^{c}$ can be

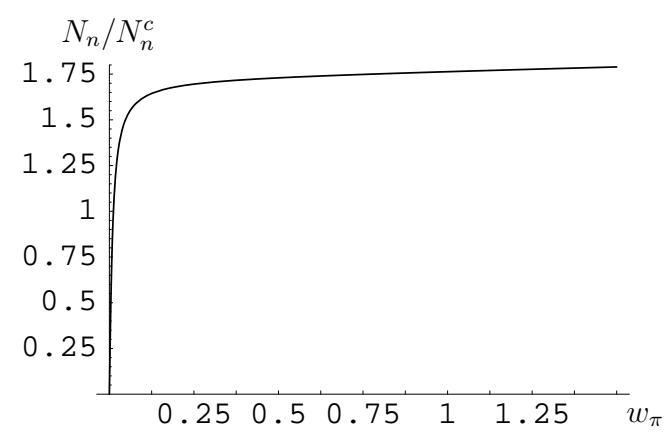

Figure 6: The ratio $N_{n} / N_{n}^{c}$ as a function $w_{\pi}$ for dominantly $\phi^{c}$ mode in the case of $|c| \geq 1 / 2$. We use $c=0.5$ and $n=10$.

approximated by

$$
\left|\frac{N_{n}}{N_{n}^{c}}\right| \approx\left|\frac{n \pi w_{\pi}}{c(1-c)}\right| .
$$

The result in this case represents $\mathcal{O}\left(w_{\pi}\right)$ mixing of $b_{n}^{c}$ characteristic of the perturbation between nondegenerate states. Using (3.36) for $w_{\pi} \approx \mathcal{O}(1)$, we obtain a wave function with the $\mathcal{O}(1)$ mixing between $\phi$ and $\phi^{c}$ components

$$
\left|\frac{N_{n}}{N_{n}^{c}}\right| \approx \sqrt{3},
$$

as anticipated by the $w_{\pi}$ perturbation between the nearly degenerate $\left(w_{\pi} \gg 1 / n \pi\right)$ states.

Dominantly $\phi^{c}$ mode: $\phi^{I=2} \equiv \phi^{c}$ 
Similarly we can obtain the dominantly $\phi^{c}$ modes corresponding to the choice of lower sign in Eq.(3.28). It is now more convenient to use the ratio

$$
\frac{N_{n}^{c}}{N_{n}}=-\frac{M_{21}}{M_{22}}
$$

$$
\frac{M_{21}}{M_{22}}=\frac{w_{\pi}}{6} \times
$$

$\frac{\left(c^{2}-c+11\right) \tan \left(x-b+\frac{\pi}{2}\right)-2 x}{c(1-c)\left(\tan \left(x-b+\frac{\pi}{2}\right) \cos (a-b)-\sin (a-b)\right)+2 x\left(\cos (a-b)+\tan \left(x-b+\frac{\pi}{2}\right) \sin (a-b)\right)}$.

For $|c|<1 / 2$ case, we show $N_{n}^{c} / N_{n}$ as a function of $w_{\pi}$ in Fig[7, using the solution (3.28) with the lower sign for the dominantly $\phi^{c}$ modes. Using (3.38) for small $w_{\pi}$, the

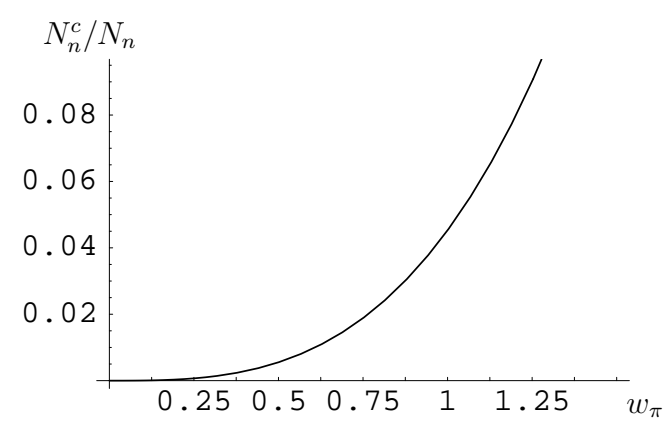

Figure 7: The ratio $N_{n}^{c} / N_{n}$ as a function $w_{\pi}$ for dominantly $\phi^{c}$ mode in the case of $|c|<1 / 2$. We use $c=0.1$ and $n=10$.

ratio is approximately given by

$$
\frac{N_{n}^{c}}{N_{n}} \approx \frac{w_{\pi}}{6 \cos c \pi}
$$

This result exhibits $\mathcal{O}\left(w_{\pi}\right)$ mixing of $\phi$ for the $w_{\pi}$ perturbation between nondegenerate states.

For $|c| \geq 1 / 2$, we show $N_{n}^{c} / N_{n}$ as a function of $w_{\pi}$ in Fig 8 , using the solution (3.28) with the lower sign for the dominantly $\phi^{c}$ modes. Using (3.35) for $w_{\pi} \ll 1 /(n \pi)$, the ratio

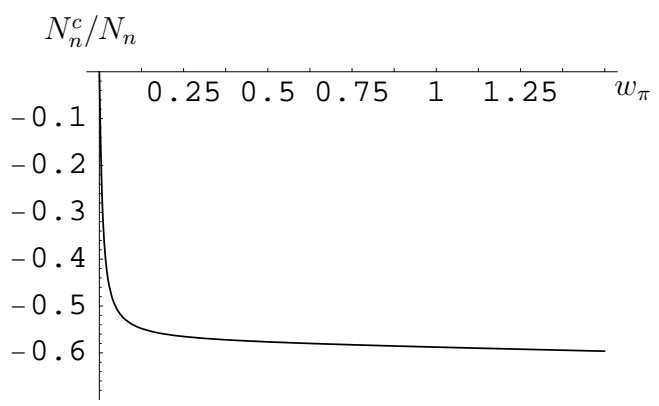

Figure 8: The ratio $N_{n}^{c} / N_{n}$ as a function $w_{\pi}$ for dominantly $\phi^{c}$ mode in the case of $|c| \geq 1 / 2$. We use $c=0.5$ and $n=10$. 
is approximately given by

$$
\left|\frac{N_{n}^{c}}{N_{n}}\right| \approx\left|\frac{n \pi w_{\pi}}{3 c(1-c)}\right|,
$$

which exhibits $\mathcal{O}\left(w_{\pi}\right)$ mixing of $\phi$ as a $w_{\pi}$ perturbation of nondegenerate states. Using (3.36) for $w_{\pi} \sim \mathcal{O}(1)$, we obtain $\mathcal{O}(1)$ mixing between $\phi$ and $\phi^{c}$ components

$$
\left|\frac{N_{n}^{c}}{N_{n}}\right| \approx \frac{1}{\sqrt{3}},
$$

as anticipated by the $w_{\pi}$ perturbation between the nearly degenerate $\left(w_{\pi} \gg 1 / n \pi\right)$ states.

\section{Comment on the radius stabilization}

In this section, we study the background solution for hypermultiplets and possible radius stabilization in the presence of constant boundary superpotential $W_{b}$ together with the compensator $\varphi$ and the radion $T$ supermultiplets.

As a simple and realistic approximation, we consider a perturbative treatment for small values of boundary superpotential $W_{b}$. Let us first find out a background solution for the case of vanishing boundary superpotential: $w_{0}=w_{\pi}=0$. By requiring no SUSY breaking with vanishing auxiliary fields $F=F^{c}=F_{\varphi}=F_{T}=0$ in Eqs. (2.5)-(2.8), we obtain a unique supersymmetric solution with a complex constant $N_{2}$,

$$
\begin{aligned}
& \phi=N_{2} \exp \left[\left(\frac{3}{2}-c\right) R \sigma\right] \equiv \phi_{s}, \\
& \phi^{c}=0 .
\end{aligned}
$$

The potential vanishes for any values of the radius $R$ and the complex parameter $N_{2}$. Therefore the supersymmetric solution $\phi_{s}$ has a flat directions along $\phi$, as is typical for a SUSY solution. Moreover, radius $R$ is undetermined in this $w_{0}, w_{\pi} \rightarrow 0$ limit.

For nonzero $w_{0}$ and $w_{\pi}$, we will find that supersymmetry is generically broken, and a nontrivial potential will be generated. Let us now assume $\left|w_{0}\right| \sim\left|w_{\pi}\right| \equiv w \ll 1$ and work

out perturbative solutions of the equations of motion for $\chi$ and $\chi^{c}$ as deviations from the supersymmetric solutions in Eqs. (5.1) and (5.2)

$$
\begin{aligned}
& \phi=\phi_{s}+\chi, \\
& \phi^{c}=\hat{\epsilon} \chi^{c},
\end{aligned}
$$

where we allow possible discontinuities of the $Z_{2}$ odd field $\phi^{c}$ across the fixed points $y=0, \pi$, similarly to Eq. (3.1). The equations of motion from the Lagrangian (2.4) shows that $\phi^{c \dagger}$ arises in the first order in $w$

$$
\begin{aligned}
& {\left[\partial_{y}+\left(\frac{3}{2}+c\right) R \sigma^{\prime}\right]\left(e^{-4 R \sigma}\left(\frac{\phi_{s}^{\dagger} \phi_{s}}{6 M_{5}^{3}}-1\right)\left[-\partial_{y}+\left(\frac{3}{2}+c\right) R \sigma^{\prime}\right] \hat{\epsilon} \chi^{c}\right)} \\
& +e^{-4 R \sigma} \frac{\phi_{s} \phi_{s}^{\dagger}}{\phi_{s}^{\dagger} \phi_{s}-6 M_{5}^{3}}(3-2 c)^{2}\left(R \sigma^{\prime}\right)^{2} \hat{\epsilon} \chi^{c}-\frac{e^{-4 R \sigma}}{2 M_{5}^{3}} \phi_{s}^{\dagger}\left(\partial_{y} W_{b}\right)=0 .
\end{aligned}
$$

Provided the bulk fermion mass parameter $c \neq 3 / 2$, we can change a variable from $y$ to a dimensionless variable $X \equiv \phi_{s}^{\dagger} \phi_{s} /\left(6 M_{5}^{3}\right)-1$, and obtain the field equation for the 
bulk $(y \neq 0, \pi)$

$$
\begin{aligned}
& {\left[-(3-2 c)^{2} X^{2}(X+1)^{2} \frac{\partial^{2}}{\partial X^{2}}\right.} \\
& -(3-2 c) X(X+1)(3-2 c+2(1-2 c) X) \frac{\partial}{\partial X} \\
& \left.+(3-2 c)^{2}+(3-2 c)(9 / 2-c) X+(1 / 2-c)(3 / 2+c) X^{2}\right] \chi^{c}=0,
\end{aligned}
$$

whose solution is given for generic values of the bulk fermion mass parameter $c(\neq 3 / 2,1 / 2)$ as

$$
\chi^{c}=X^{-1}(X+1)^{(5 / 2-c) /(3-2 c)}\left[c_{1}+c_{2}(X+1)^{-(1-2 c) /(3-2 c)}(X+(3-2 c) /(1-2 c))\right],
$$

where $c_{1}$ and $c_{2}$ are constants of integration. The solution for $c=1 / 2$ is given as

$$
\chi^{c}=X^{-1}(X+1)\left[c_{1}^{\prime}+c_{2}^{\prime}(X-\ln (X+1))\right]
$$

with another integration constants $c_{1}^{\prime}, c_{2}^{\prime}$. The field equation also yields the boundary conditions as the cancellation conditions of $\partial_{y} \delta(y)$ and $\partial_{y} \delta(y-\pi)$

$$
X\left(-\partial_{y}^{2} \hat{\epsilon}\right) \chi^{c}-\frac{1}{2 M_{5}^{3}} \phi_{s}^{\dagger}\left(\partial_{y} W_{b}\right)=0
$$

respectively. Using $W_{b}$ in Eq.(2.3) and the background solution $\phi_{s}$ in Eq.(5.1), we obtain the boundary conditions more explicitly

$$
\begin{aligned}
& \left.\chi^{c}\right|_{y=0}=-\left.\frac{\phi_{s}^{\dagger} w_{0}}{2 X}\right|_{y=0}=\frac{N_{2}^{\dagger} w_{0}}{2(1-\hat{N})}, \\
& \left.\chi^{c}\right|_{y=\pi}=\left.\frac{\phi_{s}^{\dagger} w_{\pi}}{2 X}\right|_{y=\pi}=\frac{N_{2}^{\dagger} w_{\pi} e^{(3 / 2-c) R k \pi}}{2\left(\hat{N} e^{(3-2 c) R k \pi}-1\right)},
\end{aligned}
$$

where we defined a dimensionless parameter $\hat{N} \equiv\left|N_{2}\right|^{2} /\left(6 M_{5}^{3}\right)$. These boundary conditions determine the integration constants $c_{1}, c_{2}$ (or $c_{1}^{\prime}, c_{2}^{\prime}$ ) in terms of the unique undetermined parameter $N_{2}$. We find for generic values of $c(\neq 1 / 2,3 / 2)$

$$
\begin{gathered}
c_{1}=-\left(\frac{N_{2}^{\dagger}}{2 \hat{N}^{\frac{5-2 c}{2(3-2 c)}}}\right) \frac{\left((1-2 c) \hat{N} e^{2 R k \pi}+2 e^{-(1-2 c) R k \pi}\right) w_{0}+((1-2 c) \hat{N}+2) w_{\pi} e^{-R k \pi}}{(1-2 c) \hat{N}\left(e^{2 R k \pi}-1\right)+2\left(e^{-(1-2 c) R k \pi}-1\right)}, \\
c_{2}=\left(\frac{N_{2}^{\dagger}}{2 \hat{N}^{\frac{3+2 c}{2(3-2 c)}}}\right) \frac{(1-2 c)\left(w_{0}+w_{\pi} e^{-R k \pi}\right)}{(1-2 c) \hat{N}\left(e^{2 R k \pi}-1\right)+2\left(e^{-(1-2 c) R k \pi}-1\right)} .
\end{gathered}
$$

whereas we find the solution for $c=1 / 2$ as

$$
\begin{aligned}
c_{1}^{\prime} & =-\left(\frac{N_{2}^{\dagger}}{2 \hat{N}}\right) \frac{\left(\hat{N} e^{2 R k \pi}-\ln \hat{N}-1-2 R k \pi\right) w_{0}+(\hat{N}-\ln \hat{N}-1) w_{\pi} e^{-R k \pi}}{\hat{N}\left(e^{2 R k \pi}-1\right)-2 R k \pi} \\
c_{2}^{\prime} & =\left(\frac{N_{2}^{\dagger}}{2 \hat{N}}\right) \frac{w_{0}+w_{\pi} e^{-R k \pi}}{\hat{N}\left(e^{2 R k \pi}-1\right)-2 R k \pi}
\end{aligned}
$$


In the case of $c=3 / 2$, we can solve Eq.(5.5) in the bulk directly and find

$$
\chi^{c}=c_{1}^{\prime \prime} e^{R \sigma}+c_{2}^{\prime \prime} e^{3 R \sigma}
$$

whose integration constants $c_{1}^{\prime \prime}, c_{2}^{\prime \prime}$ are determined by the boundary condition as

$$
\begin{aligned}
c_{1}^{\prime \prime} & =-\frac{N_{2}^{\dagger}}{2(\hat{N}-1)} e^{2 R k \pi} \frac{\left(w_{0}+w_{\pi} e^{-3 R k \pi}\right)}{e^{2 R k \pi}-1}, \\
c_{2}^{\prime \prime} & =\frac{N_{2}^{\dagger}}{2(\hat{N}-1)} \frac{\left(w_{0}+w_{\pi} e^{-R k \pi}\right)}{e^{2 R k \pi}-1} .
\end{aligned}
$$

From the equation of motion for $\phi$, we find that the other perturbation $\chi$ of the background $\phi_{s}$ is of order $\mathcal{O}\left(w^{2}\right)$, and that it is given in terms of the background $\phi_{s}$ and the first order perturbation $\chi^{c} \sim \mathcal{O}(w)$ uniquely, except for the amount of the admixture of the background solution $\phi_{s}$, which is always undetermined. Namely $\chi$ is determined in terms of $N_{2}$ without additional integration constants.

By inserting these solutions into the Lagrangian (2.4) and integrating over the extra dimension $y$, we obtain the potential as a function of the radius $R$ and the complex normalization parameter $N_{2}$

$$
\begin{gathered}
V=\frac{k}{2 M_{5}^{3}} \int_{0}^{\pi} d y\left\{-2 c_{2}^{\dagger} \hat{N}^{5 / 2-2 c+2 /(3-2 c)} e^{((3-2 c)(5 / 2-2 c)+2) R \sigma}\right. \\
\left.+\left(\frac{3}{2}+c+(3-2 c)\left(-\frac{5}{2}+2 c-\left[3\left(\hat{N} e^{(3-2 c) R \sigma}-1\right)\right]^{-1}\right)\right) \chi^{c \dagger}\right\} \frac{\phi_{s}^{\dagger} W_{b}}{2} e^{-4 R \sigma},
\end{gathered}
$$

where we should use the solution in Eq.(5.7) subject to the boundary condition (5.9) for $\chi^{c}$. By performing integration and using the boundary conditions (5.10) and (5.11), we find the potential

$$
\begin{aligned}
V= & -N_{2}^{\dagger} k c_{2}^{\dagger} \hat{N}^{5 / 2-2 c+2 /(3-2 c)}\left(w_{0}+w_{\pi} e^{\left((3-2 c)^{2}-2\right) R k \pi}\right) \\
& +\frac{\left|N_{2}\right|^{2} k w_{0}^{2}}{4(1-\hat{N})}\left(-4 c^{2}+12 c-6+\frac{3-2 c}{3(1-\hat{N})}\right) \\
& +\frac{\left|N_{2}\right|^{2} k w_{\pi}^{2} e^{-(1+2 c) R k \pi}}{4\left(\hat{N} e^{(3-2 c) R k \pi}-1\right)}\left(-4 c^{2}+12 c-6-\frac{3-2 c}{3\left(\hat{N} e^{(3-2 c) R k \pi}-1\right)}\right),
\end{aligned}
$$

where we should use $c_{2}$ in Eq.(5.13) for generic values of $c$. It should be replaced by $c_{2}^{\prime}$ in Eq.(5.15) for $c=1 / 2$. We find that $c=1 / 2$ case can be obtained as a smooth limit from $c \neq 1 / 2$. We see that the supersymmetry breaking induced by the boundary superpotential produces the potential $V$ as a nontrivial function of $N_{2}$ and $R$.

Let us now study the stabilization of the radius $R$ and the modulus $N_{2}$. For simplicity, we consider the case where $w_{\pi}=0$ and the constant $N_{2}$ is real. Namely we assume that the source of the SUSY breaking is localized on only one of the brane at $y=0$ (Planck brane). Then the potential becomes

$$
\begin{aligned}
V= & \frac{3 M_{5}^{3} k w_{0}^{2}}{2}\left\{\frac{-2(1-2 c)}{(1-2 c)\left(e^{2 R k \pi}-1\right) \hat{N}+2\left(e^{(2 c-1) R k \pi}-1\right)} \hat{N}^{4-2 c-\frac{1}{3-2 c}}\right. \\
& \left.+\frac{\hat{N}}{1-\hat{N}}\left(-4 c^{2}+12 c-6+\frac{3-2 c}{3(1-\hat{N})}\right)\right\} .
\end{aligned}
$$


We need to require the stationary condition for both modes $R$ and $N_{2}$

$$
\frac{\partial V}{\partial R}=0 \text { and } \frac{\partial V}{\partial \hat{N}}=0
$$

The former condition $\partial V / \partial R=0$ leads to

$$
\hat{N}=e^{-(3-2 c) R k \pi}
$$

whereas the latter condition gives

$$
\begin{aligned}
0= & -\frac{2\left(4-2 c-\frac{1}{3-2 c}\right) \hat{N}^{3-2 c-\frac{1}{3-2 c}}}{\hat{N}\left(e^{2 R k \pi}-1\right)-\frac{2}{2 c-1}\left(e^{(2 c-1) R k \pi}-1\right)}+\frac{2\left(e^{2 R k \pi}-1\right) \hat{N}^{4-2 c-\frac{1}{3-2 c}}}{\left[\hat{N}\left(e^{2 R k \pi}-1\right)-\frac{2}{2 c-1}\left(e^{(2 c-1) R k \pi}-1\right)\right]^{2}} \\
& +\frac{1}{(1-\hat{N})^{2}}\left(-4 c^{2}+12 c-6+\frac{(3-2 c)(1+\hat{N})}{3(1-\hat{N})}\right) .
\end{aligned}
$$

The vacuum expectation values for $\hat{N}$ and $(R k)$ are obtained as solutions of Eqs.(5.23) and (5.24), depending only on the mass parameter $c$. We find that there is a unique nontrivial minimum with a finite value of the radius $R$ and the normalization $N_{2}$ for the flat direction $\phi$ provided $c<c_{\mathrm{cr}}$ with

$$
c_{\mathrm{cr}} \equiv \frac{17-\sqrt{109}}{12} .
$$

At the critical value of the mass parameter $c_{\mathrm{cr}}$, the minimum occurs at infinite radius and vanishing normalization $N_{2}$

$$
\hat{N}\left(c_{\mathrm{cr}}\right)=0, \quad R\left(c_{\mathrm{cr}}\right)=\infty .
$$

To examine the stabilization for $c<c_{\mathrm{cr}}$ more closely, we parametrize $c=c_{\mathrm{cr}}-\Delta c$ with a small $\Delta c$. After using the relation Eq.(5.23),$\hat{N}=e^{-(3-2 c) R k \pi}$, we find that the potential (5.21) for $c=c_{\text {cr }}-\Delta c$ at the leading order of $\Delta c$ and $\hat{N}$ consists of two pieces

$$
\begin{aligned}
V & \approx \frac{3 M_{5}^{3} k w_{0}^{2}}{2}\left(V_{1}+V_{2}\right) \\
V_{1} & \equiv \frac{2\left(2 c_{\mathrm{cr}}-1\right)}{3-2 c_{\mathrm{cr}}} \hat{N}^{\frac{4 c_{\mathrm{cr}}^{2}-12 c_{\mathrm{cr}}+10}{3-2 c_{\mathrm{cr}}}} \\
V_{2} & \equiv-\hat{N}\left(-8 c_{\mathrm{cr}}+\frac{34}{3}\right) \Delta c
\end{aligned}
$$

The first piece $V_{1}$ is positive and increases with a power larger than unity as a function of $\hat{N}$, whereas the second piece is negative and linear in $\hat{N}$, whose coefficient is proportional to $\Delta c$. The potential $V$ and its pieces $V_{1}, V_{2}$ are depicted as a function of $\hat{N}$ in Fig 9. It is now obvious that a unique minimum occurs at finite values of $\hat{N}$ provided $\Delta c>0\left(c<c_{\mathrm{cr}}\right)$ and that the minimum point approaches $\hat{N} \rightarrow 0$ as $\Delta c \rightarrow 0\left(c \rightarrow c_{\mathrm{cr}}\right)$. Actually the Fig.9 demonstrates only the stability along the direction of $\hat{N}$, after the other variable $R$ is eliminated by the stationary condition (5.23). We have checked that this minimum point gives a true minimum of the potential $V(R, \hat{N})$ as a function of two variables, establishing the stability in both directions. For $\Delta c \neq 0$, the stationary condition $\partial V / \partial \hat{N}=0$ becomes

$$
0 \approx \frac{2\left(2 c_{\mathrm{cr}}-1\right)}{3-2 c_{\mathrm{cr}}}\left(3-2 c_{\mathrm{cr}}+\frac{1}{3-2 c_{\mathrm{cr}}}\right) \hat{N}^{2-2 c_{\mathrm{cr}}+\frac{1}{3-2 c_{\mathrm{cr}}}}-\left(\frac{34}{3}-8 c_{\mathrm{cr}}\right) \Delta c .
$$




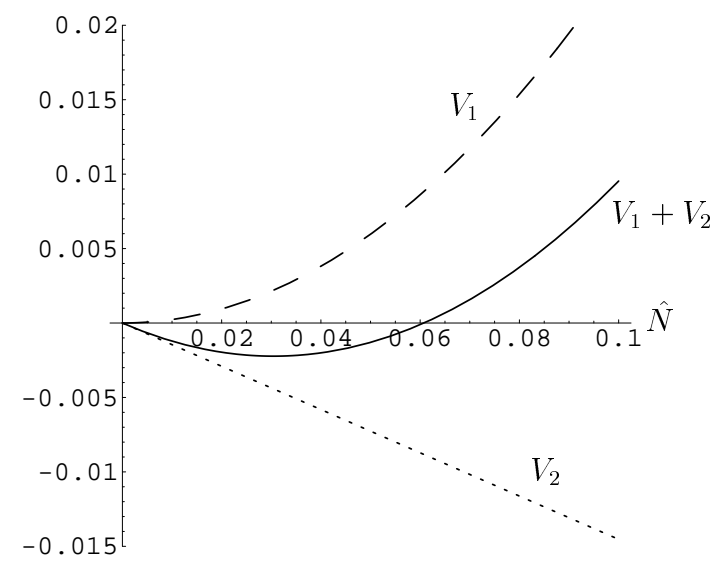

Figure 9: Potential for $c=c_{\mathrm{cr}}-\Delta c$.

From this equation, we find that the stationary point at the leading order of $\Delta c$ as

$$
\begin{aligned}
& R \approx \frac{-1}{\left[2\left(1-c_{\mathrm{cr}}\right)\left(3-2 c_{\mathrm{cr}}\right)+1\right] k \pi} \ln \left[\frac{\left(3-2 c_{\mathrm{cr}}\right)\left(\frac{17}{3}-4 c_{\mathrm{cr}}\right)}{2\left(2 c_{\mathrm{cr}}-1\right)\left(2-c_{\mathrm{cr}}-\frac{1-c_{\mathrm{cr}}}{3-2 c_{\mathrm{cr}}}\right)} \Delta c\right], \\
& \hat{N} \approx\left[\frac{\left(3-2 c_{\mathrm{cr}}\right)\left(\frac{17}{3}-4 c_{\mathrm{cr}}\right)}{2\left(2 c_{\mathrm{cr}}-1\right)\left(2-c_{\mathrm{cr}}-\frac{1-c_{\mathrm{cr}}}{3-2 c_{\mathrm{cr}}}\right)} \Delta c\right]^{\frac{3-2 c_{\mathrm{cr}}}{\left(3-2 c_{\mathrm{cr} r}\right)\left(2-2 c_{\mathrm{cr} r}\right)+1}}
\end{aligned}
$$

Numerically $R$ is evaluated as

$$
R \approx \frac{1}{10 k}\left(\ln \frac{1}{\Delta c}-3.4\right) .
$$

which implies that $R k>1$ is satisfied for $\Delta c<10^{-6}$. Therefore our model with supersymmetry breaking by constant boundary superpotentials realizes the radius stabilization at $R k>1$ without requiring any additional mechanism.

Another attempt for a possible approximation is given in Appendix $\mathbf{C}$

\section{Conclusion}

In this paper, we have studied SUSY breaking by constant superpotentials at the boundaries of the Randall-Sundrum geometry. We have calculated the Kaluza-Klein mass spectrum of hypermultiplet in the SUSY Randall-Sundrum model including constant superpotentials localized at branes. In particular, we have taken account of the bulk mass parameter $c$, radion and compensator.

Here we note that the presence of the compensator does contribute to the above calculated spectrum of hyperscalars. In the auxiliary field Lagrangian (2.9), $F$ component of the compensator gives the following type of terms

$$
W_{b} \phi \partial_{y} \phi^{c}, \quad W_{b} \phi^{c} \partial_{y} \phi, \quad W_{b} \sigma^{\prime} \phi \phi^{c}, \quad W_{b}^{2}\left(\phi^{\dagger} \phi+\phi^{c \dagger} \phi^{c}\right),
$$

and their hermitian conjugate. From (6.34), the equation of motion for $\phi$ includes $W_{b} \partial_{y} \phi^{c}, \quad W_{b}^{2} \phi^{\dagger}$, which are relevant to the boundary condition coming from the $\delta^{2}$ terms 
as in (3.9) and $W_{b} \partial_{y} \phi^{c}, \quad W_{b} \sigma^{\prime} \phi^{c}$, which are relevant to the boundary condition coming from the $\delta$ terms as in (3.11). Therefore the boundary conditions (3.15) to determine mass eigenvalues is different if we do not introduce the compensator.

In addition, we find that the SUSY breaking mass splitting and mode functions exhibit qualitatively different $w_{\pi}$ dependence for different values of the bulk mass parameter $c$.

As mentioned in Introduction, it is not yet clear what is the SS SUSY breaking in warped space. However, it has been shown that the SS SUSY breaking and SUSY breaking by the brane localized constant superpotentials are equivalent in flat space [6, 7, 8]. Therefore our results should reduce to the SS SUSY breaking if we take the flat limit. Since we have used the approximation $m_{n} / k \ll 1$ in order to focus on the effect of the warp factor, we cannot take the flat limit immediately from our results. Nevertheless it is interesting to observe the following similarity with the SS SUSY breaking in our mass spectrum. Let us first note that the bulk mass parameter $c$ should have large magnitude in order to take a proper flat limit $k \rightarrow 0$ as seen from Eq.(2.2). Our result for large $|c|$ shows a linear dependence on $w_{\pi}$, which is quite similar to that of the SS SUSY breaking as summarized in Appendix B. This similarity seems to suggest the equivalence of the SS SUSY breaking with the SUSY breaking by constant superpotentials might be extendable in some way from flat space to the warped space. This is in accord with a recent proposal that the SS mechanism works in the supergravity gauged by a $Z_{2}$ even coupling [11. On the other hand, we have found that the mass splitting depends on the bulk mass parameter for $|c|<1 / 2$, which is a new pattern of SUSY breaking. It would be interesting that the different behavior for different values of the bulk mass parameter $c$ might be understood by the analysis in the gauged supergravity. We hope that this work would give some insights to clarify whether the equivalence holds between SS breaking and SUSY breaking by constant superpotentials even in warped space.

We have also discussed the possibility of radius stabilization. In the limit of vanishing constant boundary superpotentials, we have obtained a SUSY background solutions as a solution of the classical equations of motion. The solution has two flat directions, the radius $R$ and the complex moduli parameter $N_{2}$ for the amplitude along one of the hypermultiplet scalar $\phi$. Then, we have introduced constant boundary superpotentials $w_{0, \pi} \ll 1$ as small perturbations. For the simplest situation of $w_{\pi}=0$ (boundary superpotential only for the Planck brane), we obtain the deviations from the SUSY solutions in the leading order of $w_{0}$. Using these solutions, we have explicitly calculated the radion potential, and have shown that the radius $R$ and the moduli $N_{2}$ are indeed stabilized at finite values.

When the classical solution is trivial $\phi=\phi^{c}=0$, the radion potential is generated at 1-loop after SUSY is broken and the radius might be stabilized by the Casimir energy. If SUSY is broken at high energy, the radion is stabilized for a special bulk mass parameter $c=1 / 2[25$. This mechanism might be helpful in the present case.

The radius stabilization has been studied also in the $\mathrm{AdS}_{4}$ background where SS SUSY breaking can be formulated. In models with nonzero superpotential [26], it has been found that hypermultiplets give positive contributions to the radion potential, contrary to the negative contributions from the gravity multiplet. This provides various patterns of radion potential. However explicit calculation with arbitrary values of $c$ remains to be studied. It would be interesting to apply our analysis for arbitrary values of $c$ to the radius stabilization of the $\mathrm{AdS}_{4}$ background. 


\section{Acknowledgements}

The authors thank Y. Sakamura for a useful discussion and Yuri Shirman for bringing interesting works to our attention. We also thank a referee for a valuable comment on the radius stabilization. This work is supported in part by Grant-in-Aid for Scientific Research from the Ministry of Education, Culture, Sports, Science and Technology, Japan No.17540237 (N.S.) and 16028203 for the priority area "origin of mass" (N.S. and N.U.). N.M. is supported by INFN.

\section{A Solving boundary conditions for mass spectrum}

The boundary conditions from $\delta^{2}$ terms (3.8) and (3.9) can be rewritten in terms of the Bessel functions

$$
\begin{gathered}
\frac{1}{N_{n}}\left[J_{\alpha}\left(m_{n} / k\right)+b_{\alpha}\left(m_{n}\right) Y_{\alpha}\left(m_{n} / k\right)\right]=\frac{2}{w_{0} N_{n}^{c}}\left[J_{\beta}\left(m_{n} / k\right)+b_{\beta}\left(m_{n}\right) Y_{\beta}\left(m_{n} / k\right)\right], \\
\frac{1}{N_{n}}\left[J_{\alpha}\left(m_{n} e^{R k \pi} / k\right)+b_{\alpha}\left(m_{n}\right) Y_{\alpha}\left(m_{n} e^{R k \pi} / k\right)\right] \\
=-\frac{2}{w_{\pi} N_{n}^{c}}\left[J_{\beta}\left(m_{n} e^{R k \pi} / k\right)+b_{\beta}\left(m_{n}\right) Y_{\beta}\left(m_{n} e^{R k \pi} / k\right)\right] .
\end{gathered}
$$

Similarly, the boundary conditions from $\delta$ terms (3.10) and (3.11) can be rewritten

$$
\begin{aligned}
& \left(\frac{5}{6} w_{0}^{2}-1-2 c\right)\left(J_{\alpha}\left(m_{n} / k\right)+b_{\alpha}\left(m_{n}\right) Y_{\alpha}\left(m_{n} / k\right)\right) \\
& =\frac{2 m_{n}}{k}\left(J_{\alpha}^{\prime}\left(m_{n} / k\right)+b_{\alpha}\left(m_{n}\right) Y_{\alpha}^{\prime}\left(m_{n} / k\right)\right)+\frac{1}{3} w_{0} \frac{m_{n}}{k} \frac{N_{n}}{N_{n}^{c}}\left[J_{\beta}^{\prime}\left(m_{n} / k\right)+b_{\beta}\left(m_{n}\right) Y_{\beta}^{\prime}\left(m_{n} / k\right)\right] \\
& \left(\frac{5}{6} w_{\pi}^{2}-1-2 c\right)\left(J_{\alpha}\left(m_{n} e^{R k \pi} / k\right)+b_{\alpha}\left(m_{n}\right) Y_{\alpha}\left(m_{n} e^{R k \pi} / k\right)\right) \\
& =\frac{2 m_{n}}{k} e^{R k \pi}\left(J_{\alpha}^{\prime}\left(m_{n} e^{R k \pi} / k\right)+b_{\alpha}\left(m_{n}\right) Y_{\alpha}^{\prime}\left(m_{n} e^{R k \pi} / k\right)\right) \\
& -\frac{1}{3} w_{\pi} \frac{m_{n}}{k} \frac{N_{n}}{N_{n}^{c}}\left[e^{R k \pi}\left(J_{\beta}^{\prime}\left(m_{n} e^{R k \pi} / k\right)+b_{\beta}\left(m_{n}\right) Y_{\beta}^{\prime}\left(m_{n} e^{R k \pi} / k\right)\right)\right] .
\end{aligned}
$$

We can determine $b_{\alpha}, b_{\beta}$ from the boundary conditions (A.1) and (A.3) at $y=0$

$$
\begin{aligned}
b_{\alpha}\left(m_{n}\right)= & \frac{1}{(\beta+5) w_{0}^{2}-6(1+2 c-2 \alpha)} \\
& \times\left[\left(-(\beta+5) w_{0}^{2}+6(1+2 c+2 \alpha)\right) \frac{J_{\alpha}\left(m_{n} / k\right)}{Y_{\alpha}\left(m_{n} / k\right)}+4 \beta w_{0} \frac{N_{n}}{N_{n}^{c}} \frac{J_{\beta}\left(m_{n} / k\right)}{Y_{\alpha}\left(m_{n} / k\right)}\right] \\
\sim & \frac{1}{(\beta+5) w_{0}^{2}-6(1+2 c-2 \alpha)} \\
& \times\left[\left((\beta+5) w_{0}^{2}-6(1+2 c+2 \alpha)\right)\left(\frac{m_{n}}{2 k}\right)^{2 \alpha} \frac{\pi}{\Gamma(\alpha+1) \Gamma(\alpha)}\right. \\
& \left.-4 \beta w_{0} \frac{N_{n}}{N_{n}^{c}}\left(\frac{m_{n}}{2 k}\right)^{\alpha+\beta} \frac{\pi}{\Gamma(\beta+1) \Gamma(\alpha)}\right]
\end{aligned}
$$




$$
\begin{aligned}
b_{\beta}\left(m_{n}\right)= & \frac{1}{(\beta+5) w_{0}^{2}-6(1+2 c-2 \alpha)} \\
& \times\left[12 \alpha w_{0} \frac{J_{\alpha}\left(m_{n} / k\right)}{Y_{\beta}\left(m_{n} / k\right)} \frac{N_{n}^{c}}{N_{n}}+\left((\beta-5) w_{0}^{2}+6(1+2 c-2 \alpha)\right) \frac{J_{\beta}\left(m_{n} / k\right)}{Y_{\beta}\left(m_{n} / k\right)}\right] \\
\sim & \frac{1}{(\beta+5) w_{0}^{2}-6(1+2 c-2 \alpha)} \\
& \times\left[-12 \alpha w_{0}\left(\frac{m_{n}}{2 k}\right)^{\alpha+\beta} \frac{\pi}{\Gamma(\alpha+1) \Gamma(\beta)} \frac{N_{n}^{c}}{N_{n}}\right. \\
& \left.-\left((\beta-5) w_{0}^{2}+6(1+2 c-2 \alpha)\right)\left(\frac{m_{n}}{2 k}\right)^{2 \beta} \frac{\pi}{\Gamma(\beta+1) \Gamma(\beta)}\right]
\end{aligned}
$$

where we used the approximation for $|z| \ll 1$

$$
\frac{J_{\beta}(z)}{Y_{\alpha}(z)} \sim\left(\frac{z}{2}\right)^{\beta}\left(-\frac{1}{\Gamma(\beta+1)}\right)\left(\frac{z}{2}\right)^{\alpha} \sin (\pi \alpha) \Gamma(1-\alpha)=-\left(\frac{z}{2}\right)^{\alpha+\beta} \frac{\pi}{\Gamma(\beta+1) \Gamma(\alpha)}
$$

In the approximation (3.12), we can neglect $b_{\alpha}, b_{\beta}$ since $\left(m_{n} / k\right)^{2 \alpha},\left(m_{n} / k\right)^{\alpha+\beta},\left(m_{n} / k\right)^{2 \beta}$ are very small. Using the other approximation condition (3.13), we can approximate the Bessel functions for $|z|>>1$ as

$$
\begin{aligned}
& J_{\alpha}(z) \sim \sqrt{\frac{2}{\pi z}}\left[\cos \left(z-\frac{2 \alpha+1}{4} \pi\right)-\frac{4 \alpha^{2}-1}{8 z} \sin \left(z-\frac{2 \alpha+1}{4} \pi\right)\right], \\
& Y_{\alpha}(z) \sim \sqrt{\frac{2}{\pi z}}\left[\sin \left(z-\frac{2 \alpha+1}{4} \pi\right)-\frac{4 \alpha^{2}-1}{8 z} \sin \left(z-\frac{2 \alpha+1}{4} \pi\right)\right],
\end{aligned}
$$

Therefore, it is a good approximation to impose the boundary conditions at $y=\pi$ with only $J_{\alpha(\beta)}$ terms disregarding $Y_{\alpha(\beta)}$ terms. Then, the approximated boundary conditions at $y=\pi(3.10)$ and (3.11) can be read

$$
\begin{aligned}
0 \sim & \frac{2}{N_{n}^{c}} \cos \left(\frac{m_{n}}{k} e^{R k \pi}-\frac{2 \beta+1}{4} \pi\right)+\frac{w_{\pi}}{N_{n}} \cos \left(\frac{m_{n}}{k} e^{R k \pi}-\frac{2 \alpha+1}{4} \pi\right) \\
0 \sim & \frac{1}{N_{n}}\left[c(1-c) \cos \left(\frac{m_{n}}{k} e^{R k \pi}-\frac{2 \alpha+1}{4} \pi\right)-\frac{2 m_{n}}{k} e^{R k \pi} \sin \left(\frac{m_{n}}{k} e^{R k \pi}-\frac{2 \alpha+1}{4} \pi\right)\right] \\
& +\frac{w_{\pi}}{N_{n}^{c}}\left[\frac{c^{2}-c+11}{6} \cos \left(\frac{m_{n}}{k} e^{R k \pi}-\frac{2 \beta+1}{4} \pi\right)+\frac{m_{n} e^{R k \pi}}{3 k} \sin \left(\frac{m_{n}}{k} e^{R k \pi}-\frac{2 \beta+1}{4} \pi\right)\right]
\end{aligned}
$$

which gives the matrix eigenvalue equation (3.15).

\section{B Relation to SS SUSY breaking}

In this appendix, we briefly review that the SS SUSY breaking and SUSY breaking by the brane localized constant superpotentials are equivalent in flat space [6, 7, [8] and compare our spectrum in the SUSY Randall-Sundrum model with that in flat space. Let us consider the following hypermultiplet Lagrangian in flat space

$$
\mathcal{L}=\int d^{4} \theta\left(|\Phi|^{2}+\left|\Phi^{c}\right|^{2}\right)+\left[\int d^{2} \theta \Phi^{c} \partial_{y} \Phi+\text { h.c. }\right]
$$


Its auxiliary field Lagrangian can be read as

$$
\mathcal{L}_{\text {aux }}=\left(|F|^{2}+\left|F^{c}\right|^{2}\right)+\left[F^{c} \partial_{y} \phi-F \partial_{y} \phi^{c}+\text { h.c. }\right],
$$

then, it is easy to solve

$$
F^{\dagger}=\partial_{y} \phi^{c}, \quad F^{c \dagger}=-\partial_{y} \phi .
$$

Here, we twist the hyperscalar in terms of R-symmetry rotation $S U(2)_{R}$,

$$
\left(\begin{array}{c}
\phi \\
\phi^{c \dagger}
\end{array}\right)(y+2 \pi R)=e^{2 \pi i q \sigma_{2}}\left(\begin{array}{c}
\phi \\
\phi^{c \dagger}
\end{array}\right)(y),
$$

where $q$ is $S U(2)_{R}$ charge. This can be written as

$$
\left(\begin{array}{c}
\phi \\
\phi^{c \dagger}
\end{array}\right)(y)=e^{i \sigma_{2} f(y)}\left(\begin{array}{c}
\tilde{\phi} \\
\tilde{\phi}^{c \dagger}
\end{array}\right)(y),
$$

where $\tilde{\phi}^{c}$ are periodic functions for $S^{1}$ and $f(y)$ was found in [7],

$$
f(y)=\frac{w_{0}-w_{\pi}}{4} \hat{\epsilon}(y)+\frac{w_{0}+w_{\pi}}{4} \eta(y)
$$

where

$$
\eta(y)=2 l+1, \quad l \pi R<y<(l+1) \pi R,(l: \text { integer })
$$

is the "staircase" function that jumps by two units for every $\pi R$ along $y$. Note $f(y+$ $2 \pi R)=f(y)+w_{0}+w_{\pi}$, which implies $w_{0}+w_{\pi}=2 \pi q$ to yield a correct twist. Then the equation of motion for $\tilde{\phi}$ is

$$
\left(-R^{2} \eta^{\mu \nu} \partial_{\mu} \partial_{\nu}-\partial_{y}^{2}\right) \tilde{\phi}-\partial_{y}\left(f^{\prime} \tilde{\phi}^{c \dagger}\right)-f^{\prime}\left(\partial_{y} \tilde{\phi}^{c \dagger}-f^{\prime} \tilde{\phi}\right)=0 .
$$

The equation of motion for $\tilde{\phi}^{c}$ is given by

$$
\left(-R^{2} \eta^{\mu \nu} \partial_{\mu} \partial_{\nu}-\partial_{y}^{2}\right) \tilde{\phi}^{c}+\partial_{y}\left(f^{\prime} \tilde{\phi}^{\dagger}\right)+f^{\prime}\left(\partial_{y} \tilde{\phi}^{\dagger}+f^{\prime} \tilde{\phi}^{c}\right)=0 .
$$

These equations can be solved by

$$
\begin{aligned}
& \tilde{\phi}=\cos (n y+\tilde{f}) \\
& \tilde{\phi}^{c}=\sin (n y+\tilde{f})
\end{aligned}
$$

where $\tilde{f}(y)$ is the periodic function given by

$$
\begin{aligned}
& \tilde{f}= \begin{cases}\frac{w_{0}}{2}+\frac{w_{0}+w_{\pi}}{2 \pi}(2 l \pi-y) & \text { for } 2 l \pi<y<(2 l+1) \pi \\
\frac{w_{\pi}}{2}+\frac{w_{0}+w_{\pi}}{2 \pi}((2 l+1) \pi-y) & \text { for }(2 l+1) \pi<y<2(l+1) \pi \\
0 & \text { for } y=l \pi\end{cases} \\
& \tilde{f}^{\prime}=w_{0} \delta(y)+w_{\pi} \delta(y-\pi)-\frac{w_{0}+w_{\pi}}{2 \pi} .
\end{aligned}
$$

The mass spectrum is obtained as $^{7}$

$$
m_{n}=\frac{1}{R}\left[n-\frac{w_{0}+w_{\pi}}{2 \pi}\right] .
$$

This spectrum in flat case is similar to our results (3.31) and (3.37) in the RandallSundrum model for large $|c|$ since only the scalar fields in the hypermultiplet receive the mass shift which is linear in $w_{\pi}$.

\footnotetext{
${ }^{7}$ Here, a regularization different from ours in (3.7) is used for $\delta(y) \epsilon^{2}(y)$ by taking the limit for $\epsilon(y)$ first without respecting the relation $2 \delta(y)=d \epsilon(y) / d y$. This treatment results in $\delta(y) \epsilon^{2}(y)=\delta(y)$, instead of our $\delta(y) \epsilon^{2}(y)=\delta(y) / 3$ as in (3.7).
} 


\section{Another approximation for background solution}

In this appendix, we consider another approximation to explore solutions of the equations of motion.

We see that $\mathrm{F}$ components are nonlinear in $\phi, \phi^{c}$, resulting in a difficulty to solve the equations of motion for $\phi, \phi^{c}$. To examine the leading order effects of the constant boundary superpotential, we attempt the following approximation

$$
\begin{aligned}
\phi, \phi^{c} & \sim \mathcal{O}\left(R^{-3 / 2}\right), \quad w_{0, \pi} \sim \mathcal{O}(1), \\
R^{-1} & <M_{5}, \quad R \sigma \sim \mathcal{O}(10 \pi) .
\end{aligned}
$$

Evaluating the order of magnitude of the terms in the equations of motion for auxiliary fields (2.5)-(2.8), the dominant terms are found to be

$$
\begin{array}{rll}
F & \approx-\frac{e^{-R \sigma}}{R}\left[-\partial_{y} \phi^{c \dagger}+\left(\frac{3}{2}+c\right) R \sigma^{\prime} \phi^{c \dagger}+\frac{\phi}{2 M_{5}^{3}} W_{b}\right], & \text { (C.3) } \\
F^{c} & \approx-\frac{e^{-R \sigma}}{R}\left[\partial_{y} \phi^{\dagger}-\left(\frac{3}{2}-c\right) R \sigma^{\prime} \phi^{\dagger}+\frac{\phi^{c}}{2 M_{5}^{3}} W_{b}\right], & \\
F_{\varphi} & \approx-\frac{e^{-R \sigma}}{R}\left[-\frac{1}{6 M_{5}^{3}} \phi^{\dagger} \partial_{y} \phi^{c \dagger}+\frac{1-6 R \sigma}{6 M_{5}^{3}} \phi^{c \dagger} \partial_{y} \phi^{\dagger}+\frac{3(1+R \sigma)-2 R \sigma c}{6 M_{5}^{3}} \phi^{\dagger} \phi^{c \dagger} R \sigma^{\prime}-\frac{R \sigma}{M_{5}^{3}} W_{b}\right], \\
F_{T} & \approx e^{-R \sigma}\left[\frac{1}{6 M_{5}^{3}}\left\{6 \phi^{c \dagger} \partial_{y} \phi^{\dagger}-2 \phi^{c \dagger} \phi^{\dagger}\left(\frac{3}{2}-c\right) R \sigma^{\prime}+\frac{W_{b}}{M_{5}^{3}}\left(\phi^{\dagger} \phi+\phi^{c \dagger} \phi^{c}\right)\right\}+\frac{W_{b}}{M_{5}^{3}}\right] . & \text { (C.5) }
\end{array}
$$

Then we find that the auxiliary fields Lagrangian is exactly given by the bilinear Lagrangian (2.9). Let us look for the classical solution for the background because the radion potential can be obtained from the classical solution. We assume the classical solution for the background to depend only on $y$. Since we obtain the bilinear Lagrangian (2.9), the classical equations of motion is identical to the linearized one in (3.2) and (3.3), except that $\partial_{\mu} \partial_{\nu}$ term in the last lines should be discarded.

We can easily solve the equations of motion in the bulk

$$
\begin{aligned}
\phi & =e^{(3 / 2-c) R \sigma}\left[N_{1} e^{(1+2 c) R \sigma}+N_{2}\right], \\
h^{c} & =e^{(3 / 2+c) R \sigma}\left[N_{1}^{c} e^{(1-2 c) R \sigma}+N_{2}^{c}\right],
\end{aligned}
$$

where $N_{1,2}, N_{1,2}^{c}$ are integration constants. The boundary conditions from $\delta^{2}$ terms (3.8) and (3.9) become

$$
\begin{aligned}
& 0=-2\left(N_{1}^{c}+N_{2}^{c}\right)+w_{0}\left(N_{1}+N_{2}\right) \\
& 0=2 e^{(3 / 2+c) R k \pi}\left(N_{1}^{c} e^{(1-2 c) R k \pi}+N_{2}^{c}\right)+w_{\pi} e^{(3 / 2-c) R k \pi}\left(N_{1} e^{(1+2 c) R k \pi}+N_{2}\right) .
\end{aligned}
$$

The boundary conditions from $\delta$ terms (3.10) and (3.11) become

$$
\begin{aligned}
0= & -w_{0}\left[\frac{1}{3}\left(\frac{3}{2}+c\right)\left(N_{1}^{c}+N_{2}^{c}\right)+(1-2 c) \frac{1}{3} N_{1}^{c}\right] \\
& +\frac{7}{3} w_{0}\left(N_{1}^{c}+N_{2}^{c}\right)-2 N_{1}(1+2 c) \\
0= & -w_{\pi}\left[\frac{1}{3}\left(\frac{3}{2}+c\right)\left(N_{1}^{c} e^{(1-2 c) R k \pi}+N_{2}^{c}\right)+(1-2 c) \frac{1}{3} N_{1}^{c} e^{(1-2 c) R k \pi}\right] \\
& +\frac{7}{3}\left(N_{1}^{c} e^{(1-2 c) R k \pi}+N_{2}^{c}\right)+2 N_{1}(1+2 c) e^{R k \pi} .
\end{aligned}
$$


From (C.11) and (C.12), we obtain

$$
\begin{aligned}
\left(\begin{array}{c}
N_{1}^{c} \\
N_{2}^{c}
\end{array}\right)= & N_{2} w_{0}\left[-w_{0}^{2} w_{\pi} \frac{1-e^{(1-2 c) R k \pi}}{12(1+2 c)}+\frac{w_{\pi}+w_{0} e^{R k \pi}}{9 / 2+c}-\frac{w_{\pi} e^{(1-2 c) R k \pi}+w_{0} e^{R k \pi}}{11 / 2-c}\right]^{-1} \\
& \times\left(\begin{array}{c}
\left(w_{\pi}+w_{0} e^{R k \pi}\right) /(9+2 c) \\
-\left(w_{\pi} e^{(1-2 c) R k \pi}+w_{0} e^{R k \pi}\right) /(11-2 c)
\end{array}\right)
\end{aligned}
$$

From (C.9) and (C.13), we obtain

$$
\begin{aligned}
N_{1}= & N_{2} w_{0}\left[-w_{0}^{2} w_{\pi} \frac{1-e^{(1-2 c) R k \pi}}{12(1+2 c)}+\frac{w_{\pi}+w_{0} e^{R k \pi}}{9 / 2+c}-\frac{w_{\pi} e^{(1-2 c) R k \pi}+w_{0} e^{R k \pi}}{11 / 2-c}\right]^{-1} \\
& \times w_{0} w_{\pi}\left(1-e^{(1-2 c) R k \pi}\right) /(12(1+2 c)) .
\end{aligned}
$$

It is interesting to see that $N_{1}^{c}, N_{2}^{c}, N_{1} \rightarrow 0$ for $w_{0} \rightarrow 0$. We observe that the overall constant $N_{2}$ remains undetermined, because of the bilinear Lagrangian in our approximation. Moreover, we do not have good reasons to assume this constant $N_{2}$ not to depend on the radius $R$, since all these normalization constants $N_{1}, N_{2}, N_{1}^{c}, N_{2}^{c}$ are related by the boundary condition involving $R$. Therefore we cannot give a definite answer on the radius stabilization in the present approximation.

If we take into account higher order corrections and/or additional mechanisms, we can presumably determine the normalization constant, although it is a very hard task to analyze.

\section{References}

[1] S. Dimopoulos and H. Georgi, Nucl. Phys. B 193, 150 (1981); N. Sakai, Z. Phys. C 11, 153 (1981); E. Witten, Nucl. Phys. B 188, 513 (1981); S. Dimopoulos, S. Raby and F. Wilczek, Phys. Rev. D 24, 1681 (1981).

[2] N. Arkani-Hamed, S. Dimopoulos and G. R. Dvali, Phys. Lett. B 429, 263 (1998); I. Antoniadis, N. Arkani-Hamed, S. Dimopoulos and G. R. Dvali, Phys. Lett. B 436, 257 (1998).

[3] D. Cremades, L. E. Ibanez and F. Marchesano, Nucl. Phys. B 643, 93 (2002); C. Kokorelis, Nucl. Phys. B 677, 115 (2004).

[4] L. Randall and R. Sundrum, Phys. Rev. Lett. 83, 3370 (1999).

[5] J. Scherk and J. H. Schwarz, Phys. Lett. B 82, 60 (1979); Nucl. Phys. B 153, 61 (1979).

[6] D. Marti and A. Pomarol, Phys. Rev. D 64, 105025 (2001).

[7] J. A. Bagger, F. Feruglio and F. Zwirner, Phys. Rev. Lett. 88, 101601 (2002); JHEP 0202, 010 (2002); C. Biggio, F. Feruglio, A. Wulzer and F. Zwirner, JHEP 0211, 013 (2002). 
[8] T. Gherghetta and A. Riotto, Nucl. Phys. B 623, 97 (2002).

[9] L. J. Hall, Y. Nomura, T. Okui and S. J. Oliver, Nucl. Phys. B 677, 87 (2004).

[10] J. Bagger and D. V. Belyaev, JHEP 0306, 013 (2003).

[11] H. Abe and Y. Sakamura, JHEP 0602, 014 (2006).

[12] R. Altendorfer, J. Bagger and D. Nemeschansky, Phys. Rev. D 63, 125025 (2001);

J. Bagger, D. Nemeschansky and R. J. Zhang, JHEP 0108, 057 (2001).

[13] T. Gherghetta and A. Pomarol, Nucl. Phys. B 586, 141 (2000); Nucl. Phys. B 602, 3 (2001).

[14] A. Falkowski, Z. Lalak and S. Pokorski, Phys. Lett. B 491, 172 (2000).

[15] E. Bergshoeff, R. Kallosh and A. Van Proeyen, JHEP 0010, 033 (2000).

[16] H. Hatanaka, T. Inami and C. S. Lim, Mod. Phys. Lett. A 13, 2601 (1998).

[17] N. Haba, Y. Hosotani and Y. Kawamura, Prog. Theor. Phys. 111, 265 (2004).

[18] K. y. Oda and A. Weiler, Phys. Lett. B 606, 408 (2005).

[19] K. Agashe, R. Contino and A. Pomarol, Nucl. Phys. B 719, 165 (2005).

[20] Y. Hosotani, S. Noda, Y. Sakamura and S. Shimasaki, arXiv:hep-ph/0601241.

[21] N. Maru and N. Okada, arXiv:hep-th/0508113.

[22] N. Maru and N. Okada, Phys. Rev. D 70, 025002 (2004).

[23] M. Eto, N. Maru and N. Sakai, Phys. Rev. D 70, 086002 (2004).

[24] H. Abe and Y. Sakamura, JHEP 0410, 013 (2004).

[25] J. Garriga and A. Pomarol, Phys. Lett. B 560, 91 (2003).

[26] A. Katz, Y. Shadmi and Y. Shirman, arXiv:hep-th/0601036; A. Katz, M. Redi, Y. Shadmi and Y. Shirman, arXiv:hep-th/0512246. 